\title{
Improved Enhancement Scheme Using a RBFN Detector for Impulse Noise
}

\author{
Subrajeet Mohapatra, Ratnakar Dash, Pankaj Kumar Sa and Banshidhar Majhi \\ National Institute of Technology Rourkela, India \\ subrajeets@gmail.com
}

\begin{abstract}
Since noise smoothing and image enhancement are conflicting objectives in most image processing application, so it has to be dealt properly to preserve the quality of the image. The perceptual appearance of an image may be significantly improved by modifying the high frequency components to have better edge and detail information in the image. A simple method has been proposed to improve the pitfalls of the existing unsharp mask technique which removes impulsive noise while preserving the edges and provides better contrast for visual perception. Comparative analysis on standard images at different noise conditions shows that the proposed scheme, in general, outperforms the existing schemes.
\end{abstract}

\section{Introduction}

Image enhancement algorithms are used to increase the visual perception of images for specific applications. These can be classified into intensity based techniques and feature based techniques. Intensity based techniques can be modeled using the relation:

$$
I^{\prime}(x, y)=f(I(x, y))
$$

where $I(x, y)$ is the original image, $I^{\prime}(x, y)$ is the output image after enhancement, and $f$ is the transformation function applied to the whole image. Contrast stretching [1], histogram equalization [1] are the popular members within this category. Whereas the objective of featured based enhancement technique is to enhance the high frequency components or the image details of a poor contrast image. This can be expressed using the relation:

$$
I^{\prime}(x, y)=L(x, y)+\lambda \cdot H(x, y)
$$

where $L$ and $H$ represents the low frequency and high frequency components respectively. $\lambda$ is the enhancement gain required for amplifying the high frequency components of the image for better perception. In Linear unsharp masking (UM) [1] technique, a high pass filtered scaled version of the original input image is added to itself to obtain an enhanced version as shown in Figure 1.

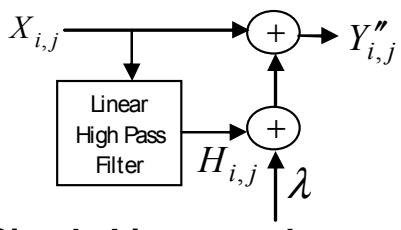

\section{Figure 1. Simple Linear unsharp masking}

In our proposed scheme we use a neural detector to determine impulse noise threshold so that we can apply selective filtering on the noisy pixels only, which prevents unnecessary loss of image details while removing impulsive noise. The proposed scheme along with the algorithm is presented in Section 2. Adaptive Threshold selection using RBFN is discussed in Section 3. Section 4 presents the simulation results and Section 5 gives the concluding remarks.

\section{Proposed Scheme}

The proposed scheme consists of impulse detection and filtration followed by image contrast enhancement as shown in Figure 2.

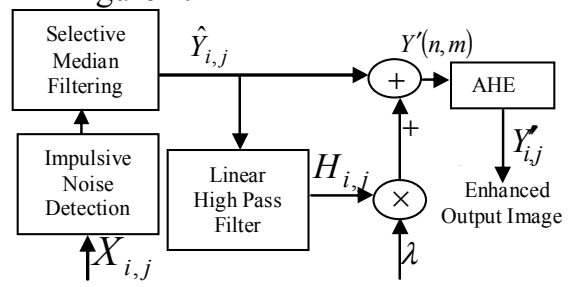

Figure 2. The proposed model

\subsection{Impulse detection}

Images are frequently contaminated by impulsive noise due to noisy sensors or channel transmission errors [1]. So the proposed scheme utilizes selective filtering before enhancement to prevent noise boosting while preserving the image details. 
Let $Y_{i, j}$ be the gray level of an original image $Y$ at pixel location $(i, j)$ and $\left[n_{\min }, n_{\max }\right]$ be the dynamic range of $Y$. Let $X_{i, j}$ be the gray level of the noisy image $X$ at pixel $(i, j)$, and then the random valued impulsive noise may be defined in (3).

$$
Y_{i, j}= \begin{cases}X_{i, j} & \text { with probability } 1-p \\ R_{i, j} & \text { with probability } p\end{cases}
$$

where $R_{i, j} \in\left[n_{\min }, n_{\max }\right]$ and $p$ is the noise ratio. Whereas for Fixed Valued Impulsive Noise (better known as Salt and Pepper Noise) $R_{i, j} \in\left\{n_{\min }, n_{\max }\right\}$. It is usually seen that removal of Salt and Pepper Noise is easier in comparison to RVIN since behavior of RVIN pixels and its surrounding pixels are very similar. In this paper we focus only on Random Valued Impulsive Noise where $Y_{i, j}$ can be of any value from $n_{\min }$ to $n_{\max }$.

In the proposed scheme, an impulsive noise detector based on second order difference is used to determine the threshold for impulse noise detection. Median filtering is performed selectively based on the decision of the threshold. The following mathematical formulation describes whether to filter or to skip a pixel located at $(i, j)$ of a test window:

$$
\hat{Y}_{i, j}= \begin{cases}Y_{i, j} & d_{i, j}=1 \\ Z_{i, j} & d_{i, j}=0\end{cases}
$$

where $Z_{i, j}=$ median $\left\{Y_{i-k, j-l},(k, l) \in W\right\}$ and $W$ is a predetermined window, usually of size $3 \times 3$ or $5 \times 5$ [3]. Filtering is performed selectively based on the decision index $d_{i, j}$ which controls the filtering operation. The algorithm is described below in two passes.

\section{Pass one:}

1. Choose a test window $X^{(T)}$ of size $3 \times 5$ centered at $(i, j)$ of $X$. Choose a sub window $X^{(W)}$ of size $3 \times 3$ centered at $(i, j)$ of $X^{(T)}$.

2. Compute the first order $3 \times 4$ difference matrix $f d$.

$$
f d_{i+k, j+l}=X_{i+k, j+l}^{(T)}-X_{i+k, j+l-1}^{(T)}
$$

Where $k=-1,0,1$ and $k=-1,0,1,2$

3. Compute the second order $3 \times 3$ difference matrix $s d$ from $f d$.

$$
s d_{i+r, j+s}=f d_{i+r, j+s+1}-f d_{i+r, j+s}
$$

Where $r=-1,0,1$ and $s=-1,0,1$

4. Compute the decision parameter $d$

$$
d_{i, j}= \begin{cases}0 & \text { if }\left|s d_{i, j}\right|>\theta_{1} \\ 1 & \text { otherwise }\end{cases}
$$

5. If $d_{i, j}$ is zero, replace the $Y_{i, j}$ pixel with the median value of its neighborhood, otherwise leave it as it is.

6. Repeat the above steps for each window from topleft to bottom-right corner of the noisy image.

\section{Pass two:}

The window $X^{(T)}$ is selected is of size $5 \times 3$ centered at $(i, j)$ of $X$ and sub window $Y^{(W)}$ of size $3 \times 3$ centered at $(i, j)$ of $X^{(T)}$. The first and second order differences are calculated in vertical fashion and the decision index is determined, followed by selective filtering similar to the steps described earlier. The threshold values taken here in this pass is $\theta_{2}$. The threshold values $\theta_{1}$ and $\theta_{2}$ are obtained using RBFN as described in Section 3.

All the steps in the second iteration are repeated for each test window column wise from top-left to bottomright corner of the image obtained from pass one. Then we perform image enhancement as described in Section 2.2.

\subsection{Image contrast enhancement}

The filtered image output $\hat{Y}_{i, j}$ is fed to a high pass filter to separate the high and low frequency components. So this can be represented using the relation given as:

$$
Y_{i, j}^{\prime}=\hat{Y}_{i, j}+\lambda H_{i, j}
$$

where $H_{i, j}$ is the output of a linear high pass filter which is obtained using relation (9).

$$
H_{i, j}=4 \hat{Y}_{i, j}-\hat{Y}_{i-1, j}-\hat{Y}_{i+1, j}-\hat{Y}_{i, j-1}-\hat{Y}_{i, j+1}
$$

where $\lambda$ is the positive gain factor that controls the level of enhancement as required by applications, which varies from one application to other. Adaptive histogram equalization (AHE) [1] technique is further applied on $Y_{i, j}^{\prime}$ for better visual perception. of the image.

\section{Adaptive threshold selection}

Artificial Neural Networks (ANN) [4] has emerged as a powerful technique to perform complex tasks in highly nonlinear dynamic environments. Numerous structural variations of ANN are available in the literature among which Radial basis functional network (RBFN) [4] is popular due to several important advantages over traditional multilayer perceptrons. The activation function used in a RBFN is usually a Gaussian function, and there are no weights connected 
between the input layer and hidden layer. In this work we use the standard Gaussian nonlinearity (basis function) as defined in (10).

$$
\phi_{i}(x)=e^{-\left\|x-c_{i}\right\|^{2} / 2 \sigma^{2}}
$$

Each Gaussian basis function consists of a centre $c_{i}$ and a variance $\sigma$ as its input parameters. The spread $(\sigma)$ of all the Gaussian basis function has been taken fixed and a standard value of 0.1 is used. The centers are randomly selected from the training sample and are used to compute $\phi_{i}$. The proposed impulsive noise detector is shown in the Figure 3, which is a twolayered structure. The input to the network is global standard deviation $\sigma$ and mean $\mu$ of the noisy image, of the image. In order to determine the error, we compare the actual output of the network with the desired output. The weight matrix between input and output layers is updated using LMS [4] algorithm depending on the error value.

We take an image say Pepper that is corrupted with impulsive noise of noise density between 0.01 and 0.30 in a step of 0.05 . Each corrupted image is subjected to the proposed filter varying the threshold from 0 to 1 in a step of 0.01 and the corresponding mean squared error (MSE) value is computed using the relation.

$$
M S E=\frac{1}{M \times N} \sum_{i=1}^{M} \sum_{j=1}^{N}\left(X_{i, j}-\hat{Y}_{i, j}\right)^{2}
$$

where $(M \times N)$ is the size of the image $X_{i, j}$ and $Y_{i, j}$, which represents the pixel values at $(i, j)^{\text {th }}$ location of original image and restored image respectively. The minimum MSE and the corresponding threshold value called optimum threshold $\left(\theta_{\text {optimum }}\right)$ are recorded. Unavailability of original image in real time forces us to use image statistics of the noisy image for threshold prediction using a RBFN (Figure3). For training the RBFN the input-output patterns $\left((\mu, \sigma) \rightarrow \theta_{\text {optimum }}\right)$ for different noise levels are generated for different images like Lena, Lisa, Boat etc. The convergence characteristic for RBFN is shown in Figure 4.

\section{Simulation results}

The superiority of the proposed scheme is demonstrated by conducting two experiments. Peak signal-to-noise ratio (PSNR) in $\mathrm{dB}$, as defined in (12) is the metric used to compare the noise removal capability of the proposed scheme with existing schemes.

$$
P S N R=10 \log _{10}\left(255^{2} / \text { MSE }\right)
$$

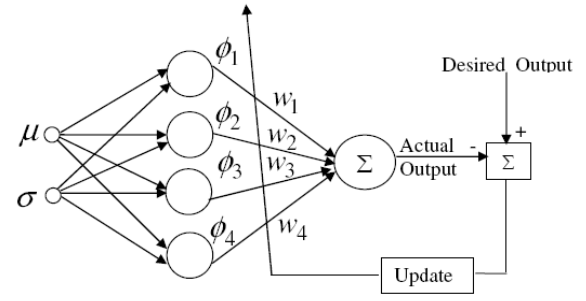

Figure 3. RBFN for threshold selection

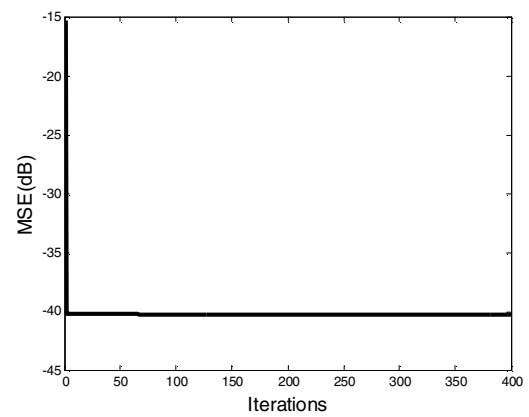

Figure 4. Convergence of RBFN

where MSE is the mean squared error as defined in (11). Subjective results for Lena, Boat and Peppers are shown in Figure6 to compare the enhancement capability.

\subsection{Experiment I}

Lena, image is corrupted with different probabilities of noise ranging from 0.01 to 0.30 . Various standard schemes like Progressive Switching Median(PSM) [5], Adaptive Center Weighted Median Filter (ACWMF) [3], Two-Pass (2-pass) [6], Switching Median $(\operatorname{SWM}(5 \times 5))[11]$, Accurate Noise Detector (AND) [7], Two-Output Nonlinear Filter (2-OUTPUT) [9], Detail Preserving Impulsive Noise removal (DPINR) [10], Median Rational Hybrid Filter-II (MRHF2) [9], Impulse Detection based on Pixel-Wise MAD(PWMAD) [12], are simulated along with the proposed scheme. PSNR obtained from various schemes for Lena image are plotted and shown in Figure5. It can be noticed from Figure 5 that the proposed noise removal scheme works better for images with $5 \%$ or more noise.

\subsection{Experiment II}

To visualize the subjective image enhancement performance, the enhanced Lena, Boat, Pepper images are compared with the results of the simple linear unsharp masking without filtering [1], linear unsharp masking after median filtering; linear unsharp masking with proposed method is shown in Figure 6. 


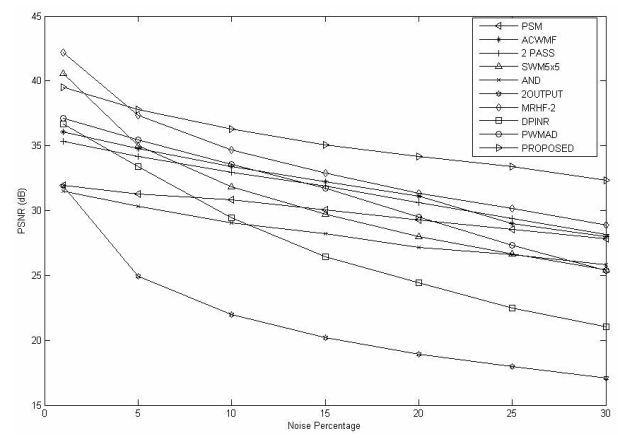

Figure 5. PSNR (dB) for Lena image

\section{Conclusion}

A novel filtering scheme has been proposed for suppressing impulsive noise from contaminated images along with provision for better image contrast. This scheme is able to preserve the image details for further image enhancement after impulse noise removal.

\section{References}

[1] R.C Gonzalez and R.E Woods, Digital Image Processing, 2nd Edition, Pearson Education

[2] D.R.K Brownrigg, The weighted median filter, Commun ACM, 27: 807- 818, 1984

[3] T.Chen, H.R Wu, Adaptive impulse detection using center weighted median filters, IEEE Sig Proc Lett, 8:13, 2001 .
[4] S.Haykin, Neural networks. $2^{\text {nd }}$ ed., Prentice-Hall.

[5] Z.Wang, D.Zhang, Progressive switching median filter for the removal of impulse noise from highly corrupted images. IEEE Trans Circuits Systems-II: Analog Digital Signal Proces , 46: 78-80, 1999

[6] X. Xu , E.L Miller, Adaptive Two-Pass Median Filter to Remove Impulsive Noise, Proc. Intl Conf on Image Processing, vol. 1, pp. I-808-I-811, 22-25, Sep. 2002

[7] K. Kondo, M. Haseyama, H,Kitajima, An accurate noise detector for image restoration, In Proc of intl conf in image processing, I-321-I-4, September 2002

[8] F. Russo, Impulse noise cancellation in image using a two-output nonlinear filters, Measurement, 36:205-13, 2004

[9] L. Khriji, M.Gabbouj, Median-rational hybrid filters. In: Proc of intl conf on image processing, p. 853-7, Oct 1998

[10] N. Alajlan, M.Kamel, E.Jernigan, Detail preserving impulsive noise removal. Sig Proc: Image Comm, 19:993-1003, 2004

[11] S. Zhang, Md Karim, A new Impulse Detector for Switching Median Filters, IEEE Sig Proc Lett, 9, 360363, Nov. 2002.

[12] V. Crnojevic, V. Senk, Z. Trpovski, Advanced impulse detection based on pixel-wise MAD, IEEE Sig Proc Lett, 11:589-92, 2004.

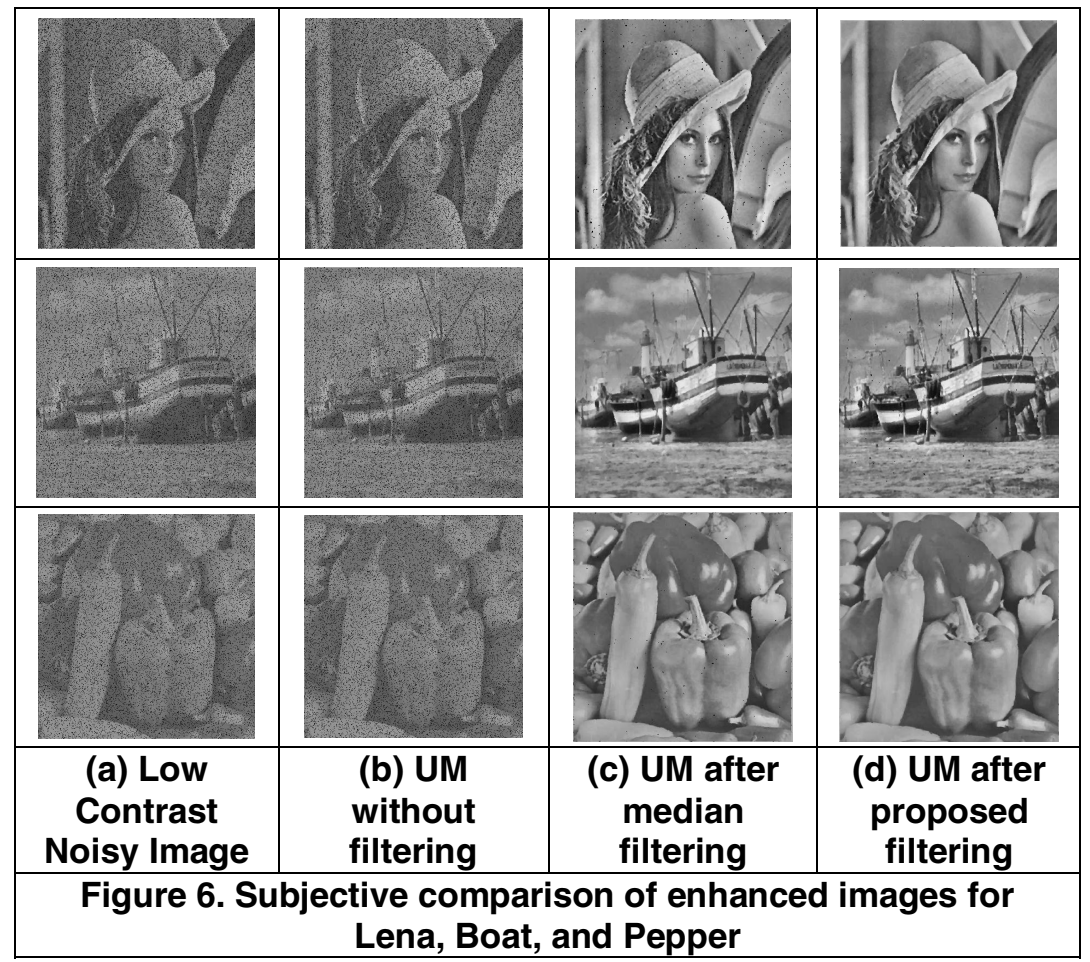

